\title{
MULTITYPE LINEAR FRACTIONAL BRANCHING PROCESSES
}

\author{
A. JOFFE, * Université de Montréal \\ G. LETAC, ${ }^{* *}$ Université Paul Sabatier
}

\begin{abstract}
We complete a paper written by Edward Pollak in 1974 on a multitype branching process the generating functions of whose birth law are fractional linear functions with the same denominator. The main tool is a parameterization of these functions adapted using the mean matrix $\boldsymbol{M}$ and an element $\boldsymbol{w}$ of the first quadrant. We use this opportunity to give a self-contained presentation of Pollak's theory.
\end{abstract}

Keywords: Linear fractional birth law; multitype branching

2000 Mathematics Subject Classification: Primary 60J80; 15A48

\section{Introduction}

Pollak (1974) developed the following idea of Harris (1963, p. 49). Suppose that we have a branching process $\left(Z_{n}\right)_{n=0}^{\infty}$ of $k$ types with birth law exemplified for $k=2$ by

$$
\begin{aligned}
& \mathrm{E}\left(s^{Z_{1}} \mid Z_{0}=(1,0)\right)=\frac{a_{11} s_{1}+a_{12} s_{2}+b_{1}}{c_{1} s_{1}+c_{2} s_{2}+d}, \\
& \mathrm{E}\left(s^{Z_{1}} \mid Z_{0}=(0,1)\right)=\frac{a_{21} s_{1}+a_{22} s_{2}+b_{2}}{c_{1} s_{1}+c_{2} s_{2}+d},
\end{aligned}
$$

where the $a_{i j}$, the $b_{i}$, the $c_{i}$, and $d$ are real numbers and $s=\left(s_{1}, s_{2}\right)$. Each function appearing in the components is called a fractional linear function, or homography. Note that the denominators are the same. Harris (1963, p. 49) observed that in such circumstances $E\left(\boldsymbol{s}_{n} \mid \boldsymbol{Z}_{0}\right)$ has a similar form. For $k=1$, when

$$
\mathrm{E}\left(s^{Z_{1}} \mid Z_{0}=1\right)=h_{G}(s)=(a s+b) /(c s+d)
$$

and when

$$
\boldsymbol{G}=\left(\begin{array}{ll}
a & b \\
c & d
\end{array}\right)
$$

is in the group $G L(2, \mathbb{R})$ of nonsingular $2 \times 2$ real matrices, elementary solutions are known for all classical problems concerning $Z_{n}$. This is due to the fact that $h_{\boldsymbol{G} \boldsymbol{G}_{1}}=h_{\boldsymbol{G}} \circ h_{\boldsymbol{G}_{1}}$ for all $\boldsymbol{G}$ and $\boldsymbol{G}_{1}$ in $G L(2, \mathbb{R})$, which implies that asymptotic calculations about $h_{\boldsymbol{G}^{n}}(s)=\mathrm{E}\left(s^{Z_{n}} \mid Z_{0}\right)$ become easy. The index of Harris (1963) gives many references to this fractional linear case. For a textbook treatment, see Toulouse (1999, Section 13.6).

Received 22 June 2006; revision received 4 September 2006.

* Postal address: Département de Mathématiques et de Statistique, Université de Montréal, C.P. 6128, succursale Centre-ville, Montréal, QC H3C 3J7, Canada.

** Postal address: Laboratoire de Statistique et Probabilités, Université Paul Sabatier, 118 route de Narbonne, 31062 Toulouse cedex, France. Email address: letac@ cict.fr 
The fact that $h_{\boldsymbol{G} \boldsymbol{G}_{1}}=h_{\boldsymbol{G}} \circ h_{\boldsymbol{G}_{1}}$ still holds for $\mathbb{R}^{k}$ is easily checked by brute calculation. The geometer will find that it hardly needs a proof, since $h_{\boldsymbol{G}}$ can be seen as a projective transformation of the projective real space of dimension $k$. Note that while there are still some restrictions on the $(k+1) \times(k+1)$ matrix $\boldsymbol{G}$, to avoid trivialities, we do not assume that $\boldsymbol{G}$ is nonsingular.

Let $\boldsymbol{M}$ be the classical mean matrix of the birth law and denote by $\boldsymbol{I}_{k}$ the unit matrix of order $k$. Pollak's exposition overlooks the fact that $\boldsymbol{I}_{k}-\boldsymbol{M}$ is not necessarily invertible when the spectral radius, $\rho$, of $M$ is larger than 1 (Equation (2.9) of Pollak (1974) and all its consequences). This note gives counterexamples (see (13), below) and corrects this point by showing that the conclusions drawn by Pollak still hold (Theorem 3, part 1). This is achieved by a systematic use of linear algebra which unifies the computations. As a by-product we obtain new results: identification of the domain of the parameters (Proposition 2); identification of the limit law of the normalized projection of $Z_{n}$ for $\rho<1$ under conditioning by $Z_{n} \neq 0$ (Theorem 1 ); identification of the limit law of the normalized projection of $Z_{n}$ on the orthogonal space of the left eigenvector $v$ of $M$ corresponding to $\rho \geq 1$, under conditioning by $Z_{n} \neq 0$ (Theorem 1 and Theorem 2, part 3); and comments on the total progeny and imbeddability (Sections 7 and 8). Basically, we present a self-contained exposition which includes most of Pollak's (1974) results. It is interesting to observe that these results can be proved without the help of the general theory of multitype branching processes.

Section 2 studies the fractional linear distributions on $\mathbb{N}^{k}$ and Section 3 studies the fractional linear birth laws extending (1) to $\mathbb{N}^{k}$. Essentially, the fractional linear birth laws are parameterized by a pair $(\boldsymbol{R}, \boldsymbol{w})$, where $\boldsymbol{R}$ is an arbitrary substochastic $k \times k$ matrix and $\boldsymbol{w}$ is an arbitrary line vector of $(0, \infty)^{k}$. Another useful parameterization is given by the pair $(\boldsymbol{M}, \boldsymbol{w})$. In particular, the $n$th iteration of the birth law can be easily expressed using the parameterization $\left(\boldsymbol{M}^{n}, \boldsymbol{w}^{(n)}\right)$ where

$$
\boldsymbol{w}^{(n)}=\boldsymbol{w}\left(\boldsymbol{I}_{k}+\boldsymbol{M}+\cdots+\boldsymbol{M}^{n-1}\right)
$$

(Proposition 3). However, the knowledge of $\boldsymbol{M}$ and $\boldsymbol{w}$ will impose strong restrictions on the domains of $\boldsymbol{w}$ and, respectively, $\boldsymbol{M}$. In particular, the matrix $\boldsymbol{M}$ of a fractional birth law is by no means arbitrary, and its row vectors have to be close to proportionality. A consequence is the surprising result (Proposition 5) that if $\rho>1$ then all the other eigenvalues of $\boldsymbol{M}$ have a modulus less than or equal to 1 . This is in contrast with the case $k=1$, where the linear fractional birth law yields the same asymptotic behavior as the general case when the variance is finite.

Sections 4, 5, and 6 give probabilistic applications of these results for $\rho<1, \rho=1$, and, respectively, $\rho>1$. Sections 7 and 8 comment on the total progeny and the imbeddability. In spite of its limitations, the multitype linear fractional process is the only one for which we can perform extensive explicit computations.

\section{Fractional linear distributions on $\mathbb{N}^{k}$}

Let us denote by $E$ the linear space of row vectors, $\boldsymbol{x}=\left(x_{1}, \ldots, x_{k}\right)$, of $k$ real numbers and by $E^{*}$ the linear space of column vectors of $k$ real numbers. Such notation distinguishes the state space $E$ from the phase space $E^{*}$. If $\boldsymbol{x}$ is in $E$ and $\boldsymbol{y}$ is in $E^{*}$ then we write $\langle\boldsymbol{x}, \boldsymbol{y}\rangle=$ $x_{1} y_{1}+\cdots+x_{k} y_{k}$, which identifies $E^{*}$ with the dual space of $E$. Note that no Euclidean structure is necessary here. A consequence of this formalism is that any $k \times k$ real matrix can be interpreted either as an endomorphism of $E^{*}$ or as an endomorphism of $E$. In particular, for $\boldsymbol{t} \in E$ and $\boldsymbol{b} \in E^{*}$ we frequently consider the square matrix $\boldsymbol{b} \otimes \boldsymbol{t}$, which represents the 
respective endomorphisms of $E$ and $E^{*}$ defined by $\boldsymbol{x} \mapsto\langle\boldsymbol{x}, \boldsymbol{b}\rangle \boldsymbol{t}$ and $\boldsymbol{y} \mapsto \boldsymbol{b}\langle\boldsymbol{t}, \boldsymbol{y}\rangle$. Note that in fact $\langle\boldsymbol{t}, \boldsymbol{b}\rangle=\boldsymbol{t} \boldsymbol{b}$ and $\boldsymbol{b} \otimes \boldsymbol{t}=\boldsymbol{b} \boldsymbol{t}$, but we believe that this less simple notation is easier to follow. Finally, sometimes we shall consider $E^{*}$ (but not $E$ ) to be a commutative algebra by defining $s \boldsymbol{s}^{\prime}$ by coordinatewise multiplication. This gives meaning to the map $s \mapsto \mathrm{e}^{s}$ from $E^{*}$ to $E^{*}$.

A fractional linear distribution on $\mathbb{N}^{k}$ is the distribution of a random variable of $E$ denoted $\boldsymbol{X}=\left(X_{1}, \ldots, X_{k}\right)$, with values in $\mathbb{N}^{k}$, such that its generating function, $f_{\boldsymbol{X}}$, has the form

$$
f_{X}\left(s_{1}, \ldots, s_{k}\right)=\mathrm{E}\left(s_{1}^{X_{1}} \cdots s_{k}^{X_{k}}\right)=\frac{a_{1} s_{1}+\cdots+a_{k} s_{k}+b}{c_{1} s_{1}+\cdots+c_{k} s_{k}+d},
$$

where $\boldsymbol{a}=\left(a_{1}, \ldots, a_{k}\right)$ and $\boldsymbol{c}=\left(c_{1}, \ldots, c_{k}\right)$ are in $E, b$ and $d$ are in $\mathbb{R}$, and $\boldsymbol{s} \in E^{*}$. We also use the compact notation

$$
f_{\boldsymbol{X}}(\boldsymbol{s})=\mathrm{E}\left(\boldsymbol{s}^{\boldsymbol{X}}\right)=\frac{\langle\boldsymbol{a}, \boldsymbol{s}\rangle+b}{\langle\boldsymbol{c}, \boldsymbol{s}\rangle+d} .
$$

Let us first observe some obvious facts about such a distribution. The sequence $(\boldsymbol{a}, b, \boldsymbol{c}, d)$ is called the sequence of parameters of the fractional linear distribution.

1. $a_{1}+\cdots+a_{k}+b=c_{1}+\cdots+c_{k}+d$, since $f_{X}(\mathbf{1})=1$.

2. Replacing $(\boldsymbol{a}, b, \boldsymbol{c}, d)$ by $(\lambda \boldsymbol{a}, \lambda b, \lambda \boldsymbol{c}, \lambda d)$ for some $\lambda \neq 0$ produces the same fractional linear distribution.

3. If $c_{i}=0$ for some $i$ then $X_{i}$ is concentrated on $\{0,1\}$.

4. In the case $d=0$ we must have $b=0$ in order to have analyticity at 0 . However, this also implies that $\boldsymbol{a}$ and $\boldsymbol{c}$ are equal, that is, $\operatorname{Pr}(\boldsymbol{X}=\mathbf{0})=1$. If $d \neq 0$ then $\operatorname{Pr}(\boldsymbol{X}=\mathbf{0})=b / d$.

5. If $a_{1}=c_{1}$ then $\mathrm{E}\left(s_{1}^{X_{1}}\right)=f_{X}\left(s_{1}, 1, \ldots, 1\right)=1$. This says that $X_{1}$ is concentrated on $\{0\}$. A similar observation holds for the other $X_{i}$.

6. The vector $\boldsymbol{m}=f_{\boldsymbol{X}}^{\prime}(\mathbf{1})$ of the means equals $(\boldsymbol{a}-\boldsymbol{c}) /\left(c_{1}+\cdots+c_{k}+d\right)$. The calculation is simplified by property 1 .

Definition 1. A fractional linear distribution with parameters $(\boldsymbol{a}, b, \boldsymbol{c}, d)$ is said to be regular if $d \neq 0$ and if, for all $i=1, \ldots, k, c_{i} \neq 0$ and $a_{i} \neq c_{i}$.

Thus, regularity implies that $\boldsymbol{X}$ is not concentrated on a set $\left\{\boldsymbol{x} \in E: x_{i}=0,1\right\}$ for some $i$. We consider the two subsets $T$ and $P$ of $E$ defined by

$$
\begin{aligned}
& T=\left\{\boldsymbol{t}=\left(t_{1}, \ldots, t_{k}\right) \in(0,1)^{k}: t_{1}+\cdots+t_{k}<1\right\}, \\
& P=\left\{\boldsymbol{r}=\left(r_{1}, \ldots, r_{k}\right) \in[0,1]^{k}: 0<r_{1}+\cdots+r_{k} \leq 1\right\} .
\end{aligned}
$$

Thus, $P$ is the closure of the open set $T$ minus the point $(0, \ldots, 0)$. For $(\boldsymbol{t}, \boldsymbol{r}) \in T \times P$, we define the fractional linear law $P(\boldsymbol{t}, \boldsymbol{r})$ on $\mathbb{N}^{k}$ with generating function

$$
f(\boldsymbol{s})=r_{0}+t_{0} \frac{r_{1} s_{1}+\cdots+r_{k} s_{k}}{1-t_{1} s_{1}-\cdots-t_{k} s_{k}}=r_{0}+t_{0} \frac{\langle\boldsymbol{r}, \boldsymbol{s}\rangle}{1-\langle\boldsymbol{t}, \boldsymbol{s}\rangle},
$$

where $r_{0}=1-\left(r_{1}+\cdots+r_{k}\right)$ and $t_{0}=1-\left(t_{1}+\cdots+t_{k}\right)$. Note that the parameters $(\boldsymbol{a}, b, \boldsymbol{c}, d)$ and the mean, $\boldsymbol{m}$, of $P(\boldsymbol{t}, \boldsymbol{r})$ are

$$
\boldsymbol{a}=t_{0} \boldsymbol{r}-r_{0} \boldsymbol{t}, \quad b=r_{0}, \quad \boldsymbol{c}=-\boldsymbol{t}, \quad d=1, \quad \boldsymbol{m}=\boldsymbol{r}+\frac{1-r_{0}}{t_{0}} \boldsymbol{t} .
$$


Remark 1. Denote by $\boldsymbol{e}_{1}, \ldots, \boldsymbol{e}_{k}$ the canonical basis of $E$; thus, for each $i, \boldsymbol{e}_{i}$ is a row vector whose $i$ th component is 1 and whose other components are 0 . Given a $t \in T$, or $\boldsymbol{w}=\boldsymbol{t} / t_{0} \in$ $(0, \infty)^{k}$ with $\boldsymbol{t}=\boldsymbol{w} /(1+\langle\boldsymbol{w}, \mathbf{1}\rangle)$, the values of $\boldsymbol{m}$ are severely restricted: in fact, $\boldsymbol{m}$ is in the closed convex simplex with extreme points $\left\{\mathbf{0}, \boldsymbol{w}+\boldsymbol{e}_{1}, \ldots, \boldsymbol{w}+\boldsymbol{e}_{k}\right\}$ with the restriction $\boldsymbol{m} \neq \mathbf{0}$. Conversely, given $\boldsymbol{m} \in(0, \infty)^{k}$, the set, $C_{\boldsymbol{m}}$, of $\boldsymbol{w} \in(0, \infty)^{k}$ such that $\boldsymbol{m}=\boldsymbol{r}+\left(1-r_{0}\right) \boldsymbol{w}$ is the intersection of $(0, \infty)^{k}$ with the strip $\{\lambda \boldsymbol{m}: \lambda \geq 1\}+C$, where $C$ is the closed convex simplex with extreme points $\left\{-\boldsymbol{e}_{1}, \ldots,-\boldsymbol{e}_{k}\right\}$.

To see that $P(\boldsymbol{t}, \boldsymbol{r})$ does exist, let us introduce the geometric distribution $g_{t}$ on $\mathbb{N}^{k}$ with generating function

$$
\frac{t_{0}}{1-t_{1} s_{1}-\cdots-t_{k} s_{k}}
$$

In other words, if $g_{t}(\mathrm{~d} \boldsymbol{x})=\sum_{\boldsymbol{\alpha} \in \mathbb{N}^{k}} g_{t}(\boldsymbol{\alpha}) \delta_{\boldsymbol{\alpha}}(\mathrm{d} \boldsymbol{x})$ we have, with $n=|\boldsymbol{\alpha}|=\alpha_{1}+\cdots+\alpha_{k}$,

$$
g_{t}(\boldsymbol{\alpha})=\frac{n !}{\alpha_{1} ! \cdots \alpha_{k} !} t_{0} t_{1}^{\alpha_{1}} \cdots t_{k}^{\alpha_{k}}=\frac{n !}{\alpha !} t_{0} t^{\alpha} .
$$

The distribution $P(\boldsymbol{t}, \boldsymbol{r})$ is a mixing of a mass at $\mathbf{0}$ with the $k$ translations of $g_{t}$ by the basis vectors. More specifically, we have

$$
P(\boldsymbol{t}, \boldsymbol{r})=r_{0} \delta_{0}+\left(r_{1} \delta_{\boldsymbol{e}_{1}}+\cdots+r_{k} \delta_{\boldsymbol{e}_{k}}\right) * g_{t},
$$

where ' $*$ ' is the convolution product, $\delta_{\boldsymbol{a}}$ is the Dirac measure on $\boldsymbol{a} \in \mathbb{N}^{k}$, and $\left(\boldsymbol{e}_{1}, \ldots, \boldsymbol{e}_{k}\right)$ is the canonical basis of $\mathbb{R}^{k}$.

The next proposition shows that each regular fractional linear distribution has the form $P(\boldsymbol{t}, \boldsymbol{r})$ for some $(\boldsymbol{t}, \boldsymbol{r}) \in T \times P$.

Proposition 1. The map $(\boldsymbol{t}, \boldsymbol{r}) \mapsto P(\boldsymbol{t}, \boldsymbol{r})$ is a bijection between $T \times P$ and the set of regular fractional linear distributions on $\mathbb{N}^{k}$.

Proof. Clearly the map is one-to-one. The surjectivity is the only thing that we have to prove. Consider the generating function $f_{\boldsymbol{X}}(\boldsymbol{s})=(\langle\boldsymbol{a}, \boldsymbol{s}\rangle+b) /(\langle\boldsymbol{c}, \boldsymbol{s}\rangle+d)$ of a fixed, regular fractional linear distribution on $\mathbb{N}^{k}$. Since $d \neq 0$ and using property 3 , without loss of generality we may assume that $d=1$. This implies that $b \geq 0$, from property 4 .

Now we observe that

$$
\frac{\langle\boldsymbol{a}, \boldsymbol{s}\rangle+b}{\langle\boldsymbol{c}, \boldsymbol{s}\rangle+1}=b+\sum_{\boldsymbol{\alpha} \in \mathbb{N}^{k}} \frac{|\boldsymbol{\alpha}| !}{\boldsymbol{\alpha} !}(-\boldsymbol{c})^{\boldsymbol{\alpha}}\left(b-\sum_{j=1}^{k} \frac{a_{j}}{c_{j}} \frac{\alpha_{j}}{|\boldsymbol{\alpha}|}\right) \boldsymbol{s}^{\boldsymbol{\alpha}} .
$$

This implies that, for all $\boldsymbol{\alpha} \in \mathbb{N}^{k}$, we have

$$
(-c)^{\alpha}\left(b-\sum_{j=1}^{k} \frac{a_{j}}{c_{j}} \frac{\alpha_{j}}{|\boldsymbol{\alpha}|}\right) \geq 0 .
$$

Denoting by $S \subset \mathbb{N}^{k}$ the set of $\boldsymbol{\alpha}$ such that $b-\sum_{j=1}^{k}\left(a_{j} / c_{j}\right)\left(\alpha_{j} /|\boldsymbol{\alpha}|\right)=0$, we find that $\boldsymbol{\alpha} \in \mathbb{N}^{k} \backslash S$ implies

$$
(-c)^{\alpha}\left(b-\sum_{j=1}^{k} \frac{a_{k j}}{c_{j}} \frac{\alpha_{j}}{|\boldsymbol{\alpha}|}\right)>0 .
$$


A little thought shows that this implies $c_{j}<0$ for all $j=1, \ldots, k$. Finally, for all $\boldsymbol{\alpha} \in \mathbb{N}^{k}$ we obtain

$$
b \geq \sum_{j=1}^{k} \frac{a_{j}}{c_{j}} \frac{\alpha_{j}}{|\alpha|} .
$$

Note that the right-hand side of this inequality is a convex combination with rational coefficients $\alpha_{i} /|\boldsymbol{\alpha}|$ for the numbers $a_{j} / c_{j}$. Since this is true for all $\boldsymbol{\alpha} \in \mathbb{N}^{k}$, this is equivalent to saying that $b \geq a_{j} / c_{j}$ for all $j=1, \ldots, k$.

We now write $\boldsymbol{t}=-\boldsymbol{c}$. To see that $\boldsymbol{t} \in T$ we observe that the function

$$
z \mapsto \frac{z\left(a_{1}+\cdots+a_{k}\right)+b}{1-z\left(t_{1}+\cdots+t_{k}\right)}
$$

is analytic in the unit disc and equals 1 for $z=1$. This proves that $t \in T$. We let $t_{0}=$ $1-\left(t_{1}+\cdots+t_{k}\right)>0$ and we write $r_{0}=b$ and $\boldsymbol{r}=\left(1 / t_{0}\right)\left(\boldsymbol{a}+r_{0} t\right)$. The fact that $r_{0}+a_{j} / t_{j} \geq 0$ for all $i$ implies that $\boldsymbol{r} \in P$. To see this we write $r_{j}=\left(1 / t_{0}\right)\left(a_{j}+r_{0} t_{j}\right) \geq 0$. The fact that $r_{0} \in[0,1)$ follows from the fact that $b / d$ is the mass of the atom at the origin and is less than 1 since the distribution is regular. Thus, $r$ is in $P$.

\section{Fractional linear birth laws}

Let us consider a $(k+1) \times(k+1)$ matrix $\boldsymbol{G}$ written in four blocks,

$$
\boldsymbol{G}=\left(\begin{array}{ll}
\boldsymbol{A} & \boldsymbol{b} \\
\boldsymbol{c} & d
\end{array}\right)
$$

where $\boldsymbol{A}$ is a $k \times k$ matrix, $\boldsymbol{c}$ is in $E, \boldsymbol{b}$ is in $E^{*}$, and $d$ is a real number. We shall denote by $\mathbf{1}$ the element of $E^{*}$ whose entries all equal 1. Assume that the real number $d$ does not equal 0 and consider the fractional linear mapping $s \mapsto h_{\boldsymbol{G}}(\boldsymbol{s})$ from a neighborhood of $\mathbf{0} \in E^{*}$ into $E^{*}$ defined by

$$
h_{G}(s)=\frac{A s+b}{\langle c, s\rangle+d} .
$$

It is easily checked that the composition of two fractional linear mappings $h_{\boldsymbol{G}}$ and $h_{\boldsymbol{G}_{1}}$ satisfies $h_{\boldsymbol{G}} \circ h_{\boldsymbol{G}_{1}}(\boldsymbol{s})=h_{\boldsymbol{G G}_{1}}(\boldsymbol{s})$ in a suitable small neighborhood of $\mathbf{0}$. This implies, in particular, that iterating the function $h_{\boldsymbol{G}} n$ times gives $h_{\boldsymbol{G}} \circ h_{\boldsymbol{G}} \circ \cdots \circ h_{\boldsymbol{G}}=h_{\boldsymbol{G}^{n}}$. Note that $h_{\boldsymbol{G}}=h_{\boldsymbol{G}_{1}}$ if and only if there exists a real number $\lambda \neq 0$ such that $\boldsymbol{G}_{1}=\lambda \boldsymbol{G}$.

If $\boldsymbol{G}$ is chosen such that each component of $h_{\boldsymbol{G}}$ is the generating function of a fractional linear distribution on $\mathbb{N}^{k}$, we shall say that $h_{G}$ is the generating function of a fractional linear birth law, and we consider the multitype branching process $Z=\left(\boldsymbol{Z}_{n}\right)_{n \geq 0}$ such that

$$
\left(\mathrm{E}\left(\boldsymbol{s}^{\boldsymbol{Z}_{1}} \mid \boldsymbol{Z}_{0}=\boldsymbol{e}_{i}\right)\right)_{i=1}^{k}=h_{\boldsymbol{G}}(\boldsymbol{s}) .
$$

Property 1 , above, shows that the $(k+1)$-dimensional column vector with each component equal to 1 is an eigenvector of $\boldsymbol{G}$. As stated in the introduction, birth laws of this type are mentioned in Harris (1963, p. 49). In these circumstances we say that the matrix $\boldsymbol{G}$ is associated with this law. According to property 6 , the matrix of the means of this birth law is

$$
\boldsymbol{M}=\frac{1}{\langle\boldsymbol{c}, \mathbf{1}\rangle+d}(\boldsymbol{A}-\mathbf{1} \otimes \boldsymbol{c}) .
$$


We shall say that this fractional linear birth law is regular if each component is regular. It is easy to see that regularity implies positive regularity in the sense of Harris (1963, p. 38). Proposition 1 enables us to parameterize the set of regular fractional linear birth laws. The following statement is an immediate consequence of Proposition 1.

Proposition 2. Let $\boldsymbol{R}=\left(r_{i j}\right)$ be a $k \times k$ matrix whose rows are in $P$, and consider the vector of $E^{*}$ defined by $\boldsymbol{R}_{0}=\mathbf{1}-\boldsymbol{R} \mathbf{1}$. Let $\boldsymbol{t}$ be fixed in $T$ and let $\boldsymbol{w}=\boldsymbol{t} / t_{0} \in(0, \infty)^{k}$. Then

$$
\boldsymbol{G} \equiv \boldsymbol{G}(\boldsymbol{t}, \boldsymbol{R})=\left(\begin{array}{cc}
t_{0} \boldsymbol{R}-\boldsymbol{R}_{0} \otimes \boldsymbol{t} & \boldsymbol{R}_{0} \\
-\boldsymbol{t} & 1
\end{array}\right)
$$

is a matrix associated with a regular fractional linear birth law. Conversely, every regular fractional linear birth law has an associated matrix which is proportional to $\boldsymbol{G}(\boldsymbol{t}, \boldsymbol{R})$, for some matrix $\boldsymbol{R}$ with rows in $P$ and some $\boldsymbol{t} \in T$. Finally, the matrix of the means is

$$
M \equiv M(t, R)=R+\left(1-R_{0}\right) \otimes w=R\left(I_{k}+1 \otimes w\right) .
$$

The transformation $(\boldsymbol{t}, \boldsymbol{R}) \mapsto(\boldsymbol{w}, \boldsymbol{M})$ satisfies

$$
\boldsymbol{R}=\boldsymbol{M}\left(\boldsymbol{I}_{k}-\mathbf{1} \otimes \boldsymbol{t}\right), \quad \boldsymbol{R}_{0}=\left(\boldsymbol{I}_{k}-t_{0} \boldsymbol{M}\right) \mathbf{1}, \quad t_{0}=\frac{1}{1+\langle\boldsymbol{w}, \mathbf{1}\rangle}, \quad \boldsymbol{t}=\frac{\boldsymbol{w}}{1+\langle\boldsymbol{w}, \mathbf{1}\rangle} .
$$

The parameterization of $\boldsymbol{G}$ in terms of $\boldsymbol{M}$ and $\boldsymbol{w}$ is given by

$$
\boldsymbol{G}=\left(\begin{array}{cc}
t_{0} \boldsymbol{M}-\mathbf{1} \otimes \boldsymbol{t} & \left(\boldsymbol{I}_{k}-t_{0} \boldsymbol{M}\right) \mathbf{1} \\
-\boldsymbol{t} & 1
\end{array}\right)
$$

Proof. The only result needed in the proof is the identity

$$
\boldsymbol{I}_{k}+\mathbf{1} \otimes \boldsymbol{w}=\boldsymbol{I}_{k}+\mathbf{1} \otimes \frac{\boldsymbol{t}}{t_{0}}=\left(\boldsymbol{I}_{k}-\mathbf{1} \otimes \boldsymbol{t}\right)^{-1} .
$$

We omit the remaining details.

Remark 2. The parameterization in terms of $(\boldsymbol{t}, \boldsymbol{R}) \mapsto G(\boldsymbol{t}, \boldsymbol{R})$ is simple in the sense that it defines a bijection of our regular fractional linear birth laws with the product set $T \times P^{k}$. The parameterization in terms of the mean $\boldsymbol{M}$ and $\boldsymbol{w}=\boldsymbol{t} / t_{0}$ offers different advantages, but one should realize that, given $\boldsymbol{w}$, the set of matrices $\boldsymbol{M}$ such that there exists a birth law associated with (2) is severely restricted by Remark 1: each row $\boldsymbol{m}_{i}$ of $\boldsymbol{M}$ must belong to the simplex of $E$ with vertices $\left\{\boldsymbol{0}, \boldsymbol{w}+\boldsymbol{e}_{1}, \ldots, \boldsymbol{w}+\boldsymbol{e}_{k}\right\}$, where $\boldsymbol{w}=\boldsymbol{t} / t_{0}$. Thus, given a matrix $\boldsymbol{M}$ with positive coefficients, there exists a $t \in T$ such that there exists a birth law associated with (2) if and only $\bigcap_{i=1}^{k} C_{\boldsymbol{m}_{i}}$ is not empty (where $C_{\boldsymbol{m}} \subset(0, \infty)^{k}$ is as defined in Remark 1 and $\boldsymbol{m}_{i}$ is the $i$ th row of $\boldsymbol{M})$. For instance, for $k=2$ the matrix

$$
\boldsymbol{M}=\left(\begin{array}{cc}
1 & 10 \\
10 & 10
\end{array}\right)
$$

cannot be the matrix of means of a fractional linear birth law.

We now observe that the parameterization in terms of $(\boldsymbol{M}, \boldsymbol{w})$ leads to an easy computation of the iterates of $h_{\boldsymbol{G}}$. 
Proposition 3. Let $\boldsymbol{G}$ be a matrix associated with a regular fractional linear birth law parameterized by $\boldsymbol{t} \in T$ and by the mean $\boldsymbol{M}$. Let $\boldsymbol{w}=\boldsymbol{t} / t_{0}$. If $\boldsymbol{G}^{n}$ is associated with $\boldsymbol{t}^{(n)} \in T$ and $\boldsymbol{M}^{n}$, then $\boldsymbol{w}^{(n)}=\boldsymbol{t}^{(n)} / t_{0}^{(n)}$ in E satisfies $\boldsymbol{w}^{(n)}=\boldsymbol{w}\left(\boldsymbol{I}_{k}+\boldsymbol{M}+\cdots+\boldsymbol{M}^{n-1}\right)$. Furthermore, let $\rho$ denote the eigenvalue of $\boldsymbol{M}$ of largest modulus, and denote by $\boldsymbol{v}$ and $\boldsymbol{\mu}$ two eigenvectors of $\boldsymbol{M}$ associated with $\rho$ that are elements of $E$ and $E^{*}$, respectively, and have positive components such that $\langle\boldsymbol{v}, \boldsymbol{\mu}\rangle=1$. Then

$$
\begin{array}{ll}
\lim _{n \rightarrow \infty} \boldsymbol{w}^{(n)}=\boldsymbol{w}\left(\boldsymbol{I}_{k}-\boldsymbol{M}\right)^{-1} & \text { for } \rho<1, \\
\lim _{n \rightarrow \infty} \frac{\boldsymbol{w}^{(n)}}{n}=\langle\boldsymbol{w}, \boldsymbol{\mu}\rangle \boldsymbol{v} & \text { for } \rho=1, \\
\lim _{n \rightarrow \infty} \frac{\boldsymbol{w}^{(n)}}{\rho^{n}}=\frac{1}{\rho-1}\langle\boldsymbol{w}, \boldsymbol{\mu}\rangle \boldsymbol{v} & \text { for } \rho>1 .
\end{array}
$$

Proof. Since $h_{G^{n}}$ is the generating function of a regular fractional linear birth law with mean $\boldsymbol{M}^{n}$, by Proposition 2 there exists a $\boldsymbol{t}^{(n)} \in T$ and a real number $\lambda_{n} \neq 0$ such that

$$
\boldsymbol{G}^{n}=\lambda_{n}\left(\begin{array}{cc}
t_{0}^{(n)} \boldsymbol{M}^{n}-\mathbf{1} \otimes \boldsymbol{t}^{(n)} & \left(\boldsymbol{I}_{k}-t_{0}^{(n)} \boldsymbol{M}^{n}\right) \mathbf{1} \\
-\boldsymbol{t}^{(n)} & 1
\end{array}\right)
$$

Thus, we write $\boldsymbol{G}^{n+1}=\boldsymbol{G}^{n} \boldsymbol{G}$ and, as a consequence, obtain

$$
\begin{aligned}
\left(\begin{array}{ccc}
t_{0}^{(n+1)} & \boldsymbol{M}^{n+1}-\mathbf{1} \otimes \boldsymbol{t}^{(n+1)} & \left(\boldsymbol{I}_{k}-t_{0}^{(n+1)} \boldsymbol{M}^{n+1}\right) \mathbf{1}
\end{array}\right) & 1 \\
-\boldsymbol{t}^{(n+1)} & \frac{\lambda_{n}}{\lambda_{n+1}}\left(\begin{array}{cc}
t_{0}^{(n)} \boldsymbol{M}^{n}-\mathbf{1} \otimes \boldsymbol{t}^{(n)} & \left(\boldsymbol{I}_{k}-t_{0}^{(n)} \boldsymbol{M}^{n}\right) \mathbf{1} \\
-\boldsymbol{t}^{(n)} & 1
\end{array}\right)\left(\begin{array}{cc}
t_{0} \boldsymbol{M}-\mathbf{1} \otimes \boldsymbol{t} & \left(\boldsymbol{I}_{k}-t_{0} \boldsymbol{M}\right) \mathbf{1} \\
-\boldsymbol{t} & 1
\end{array}\right) \\
= & \frac{\lambda_{n}}{\lambda_{n+1}}\left(\begin{array}{cc}
* & * \\
-t_{0}^{(n)} \boldsymbol{t}-t_{0} \boldsymbol{t}^{(n)} \boldsymbol{M} & t_{0}^{(n)}+t_{0} \boldsymbol{t}^{(n)} \boldsymbol{M} \mathbf{1}
\end{array}\right)
\end{aligned}
$$

(where asterisks indicate terms which do not at the moment interest us). This shows that

$$
\boldsymbol{t}^{(n+1)}=\frac{t_{0}^{(n)} \boldsymbol{t}+t_{0} \boldsymbol{t}^{(n)} \boldsymbol{M}}{t_{0}^{(n)}+t_{0} \boldsymbol{t}^{(n)} \boldsymbol{M} \mathbf{1}}, \quad t_{0}^{(n+1)}=1-\boldsymbol{t}^{(n+1)} \mathbf{1}=\frac{t_{0} t_{0}^{(n)}}{t_{0}^{(n)}+t_{0} \boldsymbol{t}^{(n)} \boldsymbol{M} \mathbf{1}} .
$$

Writing $\boldsymbol{w}^{(n)}=\boldsymbol{t}^{(n)} / t_{0}^{(n)}$, we obtain $\boldsymbol{w}^{(n+1)}=\boldsymbol{w}^{(n)} \boldsymbol{M}+\boldsymbol{t} / t_{0}$, which leads to $\boldsymbol{w}^{(n)}=\boldsymbol{w}\left(\boldsymbol{I}_{k}+\right.$ $\boldsymbol{M}+\cdots+\boldsymbol{M}^{n-1}$ ) by induction on $n$. Let us also mention here that by considering the $(2,2)$ th entry of (6) we obtain $\lambda_{n+1}=\lambda_{n}\left(t_{0}^{(n)}+\boldsymbol{t}_{0} \boldsymbol{t}^{(n)} \boldsymbol{M 1}\right)$. Since $\boldsymbol{w}^{(n)}=\boldsymbol{w}\left(\boldsymbol{I}_{k}+\boldsymbol{M}+\cdots+\boldsymbol{M}^{n-1}\right)$, we obtain $\left\langle\boldsymbol{w}^{(n+1)}, \mathbf{1}\right\rangle=\boldsymbol{w}^{(n)} \boldsymbol{M} \mathbf{1}-\boldsymbol{w}$, which gives a way to compute $\lambda_{n}$ by induction. We therefore have the set of useful formulae

$$
\lambda_{n}=t_{0}^{n}\left(1+\left\langle\boldsymbol{w}^{(n)}, \mathbf{1}\right\rangle\right), \quad \boldsymbol{t}_{0}^{(n)}=\frac{\mathbf{1}}{1+\left\langle\boldsymbol{w}^{(n)}, \mathbf{1}\right\rangle}, \quad \boldsymbol{t}^{(n)}=\frac{\boldsymbol{w}^{(n)}}{1+\left\langle\boldsymbol{w}^{(n)}, \mathbf{1}\right\rangle} .
$$

Clearly, if $\rho<1$ then the matrix $\left(\boldsymbol{I}_{k}-\boldsymbol{M}\right)^{-1}$ exists and (3) holds. If $\rho \geq 1$ then we rely on the Perron-Frobenius theorem (see Harris (1963, p. 37)), which implies that

$$
\boldsymbol{M}^{n}=\rho^{n}\left(\boldsymbol{\mu} \otimes \boldsymbol{v}+\boldsymbol{Q}^{n}\right),
$$

where the largest modulus of the eigenvalues of $\boldsymbol{Q}$ is less than or equal to $r$, with $r<1$. This leads easily to (4) and (5). 
For future use it is useful to state explicitly the form of the generating function of $\boldsymbol{Z}_{n}$ in terms of $\boldsymbol{M}$ and $\boldsymbol{w}$, and two consequences of this form.

Proposition 4. Let $\boldsymbol{G}$ be a matrix associated with a regular fractional linear birth law parameterized by $\boldsymbol{w}$ and by the mean $\boldsymbol{M}$. Using the notation of Proposition 3, we have

$$
\begin{aligned}
\left(\mathrm{E}\left(\boldsymbol{s}^{Z_{n}} \mid \boldsymbol{Z}_{0}=\boldsymbol{e}_{i}\right)\right)_{i=1}^{k}=h_{\boldsymbol{G}^{n}}(\boldsymbol{s}) & =\frac{\left[\boldsymbol{M}^{n}-\mathbf{1} \otimes \boldsymbol{w}^{(n)}\right] \boldsymbol{s}+\left[\left(1+\left\langle\boldsymbol{w}^{(n)}, \mathbf{1}\right\rangle\right) \boldsymbol{I}_{k}-\boldsymbol{M}^{n}\right] \mathbf{1}}{1+\left\langle\boldsymbol{w}^{(n)}, \mathbf{1}\right\rangle-\left\langle\boldsymbol{w}^{(n)}, \boldsymbol{s}\right\rangle}, \\
h_{\boldsymbol{G}^{n}}(\boldsymbol{s})-h_{\boldsymbol{G}^{n}}(\mathbf{0}) & =\frac{\left(1+\left\langle\boldsymbol{w}^{(n)}, \mathbf{1}\right\rangle\right) \boldsymbol{M}^{n} \boldsymbol{s}-\left\langle\boldsymbol{w}^{(n)}, \boldsymbol{s}\right\rangle \boldsymbol{M}^{n} \mathbf{1}}{\left(1+\left\langle\boldsymbol{w}^{(n)}, \mathbf{1}\right\rangle\right)\left(1+\left\langle\boldsymbol{w}^{(n)}, \mathbf{1}\right\rangle-\left\langle\boldsymbol{w}^{(n)}, \boldsymbol{s}\right\rangle\right)}, \\
\mathbf{1}-h_{\boldsymbol{G}^{n}}(\mathbf{0}) & =\frac{\boldsymbol{M}^{n} \mathbf{1}}{1+\left\langle\boldsymbol{w}^{(n)}, \mathbf{1}\right\rangle} .
\end{aligned}
$$

Proof. This is just a reformulation of (2) in which $(\boldsymbol{w}, \boldsymbol{M})$ is replaced by $\left(\boldsymbol{w}^{(n)}, \boldsymbol{M}^{n}\right)$.

Now comes a surprising result, which is fundamental to the proof of part 3 of Theorem 2.

Proposition 5. Let $\boldsymbol{M}$ be the mean matrix of a regular fractional linear birth law. Then $\boldsymbol{M}$ has at most one eigenvalue of modulus greater than 1.

Proof. Let $\rho_{0}$ be any eigenvalue of $\boldsymbol{M}$ such that $1<\left|\rho_{0}\right|$. Let $\boldsymbol{f} \in E^{*}+\mathrm{i} E^{*}$ be such that $\boldsymbol{M} \boldsymbol{f}=\rho_{0} \boldsymbol{f}$ with $\boldsymbol{f} \neq \mathbf{0}$. Observe that, for all $n$,

$$
\boldsymbol{M}^{n} \boldsymbol{f}=\rho_{0}^{n} \boldsymbol{f} .
$$

From Proposition 2 there exists some substochastic matrix $\boldsymbol{R}^{(n)}$ such that

$$
\boldsymbol{M}^{n} \boldsymbol{f}=\rho_{0}^{n} \boldsymbol{f}=\boldsymbol{R}^{(n)}\left(\boldsymbol{I}_{k}+\mathbf{1} \otimes \boldsymbol{w}^{(n)}\right) \boldsymbol{f}
$$

with $\boldsymbol{w}^{(n)}=\boldsymbol{w}\left(\boldsymbol{I}_{k}+\cdots+\boldsymbol{M}^{n-1}\right)$. Therefore,

$$
\rho_{0}^{n} \boldsymbol{f}=\boldsymbol{R}^{(n)}\left(\boldsymbol{f}+\langle\boldsymbol{w}, \boldsymbol{f}\rangle\left(1+\rho_{0}+\cdots+\rho_{0}^{n-1}\right) \mathbf{1}\right) .
$$

Since $\left|\rho_{0}\right|>1$, we have

$$
\boldsymbol{f}=\rho_{0}^{-n} \boldsymbol{R}^{(n)} \boldsymbol{f}+\frac{1-\rho_{0}^{-n}}{\rho_{0}-1}\langle\boldsymbol{w}, \boldsymbol{f}\rangle \boldsymbol{R}^{(n)} \mathbf{1} .
$$

Since $\boldsymbol{R}^{(n)}$ is substochastic, $\lim _{n \rightarrow \infty} \rho_{0}^{-n} \boldsymbol{R}^{(n)}=\mathbf{0}$ and, thus, $\boldsymbol{h}=\lim _{n \rightarrow \infty} \boldsymbol{R}^{(n)} \mathbf{1}$ exists. Thus, $\boldsymbol{f}=\left[1 /\left(\rho_{0}-1\right)\right]\langle\boldsymbol{w}, \boldsymbol{f}\rangle \boldsymbol{h}$. The fact that $\boldsymbol{f} \neq \mathbf{0}$ implies that $\langle\boldsymbol{w}, \boldsymbol{f}\rangle \neq 0$. From $\langle\boldsymbol{w}, \boldsymbol{f}\rangle=\left[1 /\left(\rho_{0}-1\right)\right]\langle\boldsymbol{w}, \boldsymbol{f}\rangle\langle\boldsymbol{w}, \boldsymbol{h}\rangle$ we therefore obtain $\rho_{0}=1+\langle\boldsymbol{w}, \boldsymbol{h}\rangle$. Now, a basic remark is that $\boldsymbol{h}$ does not depend on $\rho_{0}$. Therefore, $\rho_{0}=\rho$ and the result is proved.

Remark 3. The above proof gives an expression for $\rho$, namely $\rho=1+\langle\boldsymbol{w}, \boldsymbol{h}\rangle$ with $\boldsymbol{h}=$ $\lim _{n \rightarrow \infty} \boldsymbol{R}^{(n)}$ 1. The following proposition will help us in building a counterexample to the statement of Equation (2.9) of Pollak (1974).

Proposition 6. If $\rho>1$ then 1 is an eigenvalue of $\boldsymbol{M}$ with right eigenvector $\boldsymbol{f}$ if and only if 1 is an eigenvalue of $\boldsymbol{R}$ with right eigenvector $\boldsymbol{f}$ such that $\langle\boldsymbol{w}, \boldsymbol{f}\rangle=0$. If $\boldsymbol{R}$ is a substochastic matrix with eigenvalue 1 , then the associated eigenspace $E_{1}$ has dimension greater than or equal to two. 
Proof. First, if 1 is an eigenvalue of $\boldsymbol{M}$ with eigenvector $\boldsymbol{f}$, then

$$
\boldsymbol{f}=\boldsymbol{M}^{n} \boldsymbol{f}=\boldsymbol{R}^{(n)}\left(\boldsymbol{I}_{k}+\mathbf{1} \otimes \boldsymbol{w}^{(n)}\right) \boldsymbol{f} .
$$

From Proposition 3, we obtain

$$
\boldsymbol{f}=\boldsymbol{R}^{(n)} \boldsymbol{f}+n\langle\boldsymbol{w}, \boldsymbol{f}\rangle \boldsymbol{R}^{(n)} \mathbf{1} .
$$

The representation of $\boldsymbol{R}$ in Proposition 2 yields

$$
\boldsymbol{R}^{(n)}=\boldsymbol{M}^{n}\left(\boldsymbol{I}_{k}-\mathbf{1} \otimes \frac{\boldsymbol{w}^{(n)}}{1+\left\langle\boldsymbol{w}^{(n)}, 1\right\rangle}\right) \quad \text { and } \quad \boldsymbol{R}^{(n)} \mathbf{1}=\frac{\boldsymbol{M}^{n} \mathbf{1}}{1+\left\langle\boldsymbol{w}^{(n)}, 1\right\rangle},
$$

from which it follows that

$$
\lim _{n \rightarrow \infty} \boldsymbol{R}^{(n)} \mathbf{1}=(\rho-1) \frac{\boldsymbol{\mu} \otimes \boldsymbol{v} \mathbf{1}}{\langle\boldsymbol{w}, \boldsymbol{\mu}\rangle\langle\boldsymbol{v}, \mathbf{1}\rangle}=(\rho-1) \frac{\boldsymbol{\mu}}{\langle\boldsymbol{w}, \boldsymbol{\mu}\rangle} .
$$

Hence, $\boldsymbol{R}^{(n)} \mathbf{1}$ is bounded away from $\mathbf{0}$ and it follows from (12) that $\langle\boldsymbol{w}, \boldsymbol{f}\rangle=0$ and $\boldsymbol{f}=\boldsymbol{R} \boldsymbol{f}$.

Second, the converse result is obvious (since $\boldsymbol{M} \boldsymbol{f}=\boldsymbol{R}\left(\boldsymbol{I}_{k}+\mathbf{1} \otimes \boldsymbol{w}\right) \boldsymbol{f}=\boldsymbol{R} \boldsymbol{f}=\boldsymbol{f}$ ).

Third, if $\boldsymbol{R}$ is a substochastic matrix with eigenvalue 1, then, by the Perron-Frobenius theorem, the associated eigenspace, $E_{1}$, contains a nonnegative eigenvector. By the first argument, the eigenvector $f$ of this eigenspace cannot be a multiple of a nonnegative vector. This implies that $\operatorname{dim} E_{1} \geq 2$.

Example 1. Take $k=3, t=\left(\frac{1}{5}, \frac{1}{5}, \frac{2}{5}\right)$, and, thus, $\boldsymbol{w}=(1,1,2)$, and let

$$
\boldsymbol{R}=\left(\begin{array}{lll}
0 & 1 & 0 \\
1 & 0 & 0 \\
0 & 0 & 1
\end{array}\right) \quad \text { and } \quad \boldsymbol{M}=\boldsymbol{R}\left(\boldsymbol{I}_{k}+\mathbf{1} \otimes \boldsymbol{w}\right)=\left(\begin{array}{lll}
1 & 2 & 2 \\
2 & 1 & 2 \\
1 & 1 & 3
\end{array}\right)
$$

In this case we have $\rho=5$ and the other eigenvalues of $\boldsymbol{M}$ are \pm 1 . Thus, $(\boldsymbol{I}-\boldsymbol{M})^{-1}$ does not exist, even though $\rho>1$, contradicting Equation (2.9) of Pollak (1974). An even simpler counterexample is obtained for $k=2, \boldsymbol{R}=\boldsymbol{I}_{2}$, and an arbitrary $\boldsymbol{w}$. For $w=(1,1)$, we obtain

$$
\boldsymbol{M}=\left(\begin{array}{ll}
2 & 1 \\
1 & 2
\end{array}\right) \text {. }
$$

In (1) it is obtained by taking $a_{11}=a_{22}=1, a_{12}=a_{21}=b_{1}=b_{2}=0, d=3$, and $c_{1}=c_{2}=-1$.

\section{The case $\rho<1$}

Theorem 1. Let $Z$ be a branching process governed by a regular fractional linear birth law parameterized by $\boldsymbol{t} \in T$ and by the mean $\boldsymbol{M}$. Denote by $\boldsymbol{v}$ the left eigenvector of $\boldsymbol{M}$ for the eigenvalue normalized by $\langle\boldsymbol{v}, \mathbf{1}\rangle=1$. Let us assume that $\rho<1$. Let $\boldsymbol{w}^{(\infty)}=\boldsymbol{w}\left(\boldsymbol{I}_{k}-\boldsymbol{M}\right)^{-1}$ and

$$
\boldsymbol{t}^{(\infty)}=\frac{\boldsymbol{w}^{(\infty)}}{1+\left\langle\boldsymbol{w}^{(\infty)}, \mathbf{1}\right\rangle} .
$$

Then $\lim _{n \rightarrow \infty} Z_{n}=\mathbf{0}$ almost surely. Furthermore,

$$
\lim _{n \rightarrow \infty} \mathrm{E}\left(\boldsymbol{s}^{\boldsymbol{Z}_{n}} \mid \boldsymbol{Z}_{n} \neq \mathbf{0}, \boldsymbol{Z}_{0}=\boldsymbol{e}_{i}\right)=\frac{\left\langle\boldsymbol{v}-\boldsymbol{t}^{(\infty)}, \boldsymbol{s}\right\rangle}{1-\left\langle\boldsymbol{t}^{(\infty)}, \boldsymbol{s}\right\rangle} .
$$

In particular, this does not depend on $i=1, \ldots, k$. 
Remark 4. Note that Pollak (1974, p. 454, 11. 2-6) considered the last formula to be too complicated to be presented, while our parameterization leads to a compact form.

Proof of Theorem 1. We denote by $\boldsymbol{\mu}$ the left eigenvector of $\boldsymbol{M}$ for the eigenvalue $\rho$ such that $\langle\boldsymbol{v}, \boldsymbol{\mu}\rangle=1$. Recall that each component of $\boldsymbol{\mu}$ is positive. We use

$$
\mathrm{E}\left(\boldsymbol{s}^{\boldsymbol{Z}_{n}} \mid \boldsymbol{Z}_{n} \neq 0, \boldsymbol{Z}_{0}=\boldsymbol{e}_{i}\right)=\frac{\left(h_{\boldsymbol{G}^{n}}(\boldsymbol{s})-h_{\boldsymbol{G}^{n}}(\mathbf{0})\right)_{i}}{1-\left(h_{\boldsymbol{G}^{n}}(\mathbf{0})\right)_{i}} .
$$

Equation (8) implies that $\boldsymbol{M}^{n} \boldsymbol{s} \sim \rho^{n}\langle\boldsymbol{v}, \boldsymbol{s}\rangle \boldsymbol{\mu}$ and $\boldsymbol{M}^{n} \mathbf{1} \sim \rho^{n} \boldsymbol{\mu}$. Substituting (10) and (11) into (14) and using these equivalences and the limit (3) leads to the result.

\section{The case $\rho=1$}

Theorem 2. Let $Z$ be a branching process governed by a regular fractional linear birth law parameterized by $\boldsymbol{t} \in T$ and by the mean $\boldsymbol{M}$. Let us assume that $\rho=1$. Then the following statements hold.

1. $\lim _{n \rightarrow \infty} \boldsymbol{Z}_{n}=\mathbf{0}$ almost surely.

2. The limit of the distribution of $\boldsymbol{Z}_{n} / n$ conditioned by the event $\left\{\boldsymbol{Z}_{n} \neq \mathbf{0}, \boldsymbol{Z}_{0}=\boldsymbol{e}_{i}\right\}$ exists and is an exponential distribution concentrated on the line $\mathbb{R} \boldsymbol{v}$. More specifically, its Laplace transform is $(1+\langle\boldsymbol{w}, \boldsymbol{\mu}\rangle\langle\boldsymbol{v}, \boldsymbol{s}\rangle)^{-1}$.

3. Denote by $\pi$ the canonical projection of $E$ on the quotient space $E / \mathbb{R} \boldsymbol{v}$. Then the limit, $\alpha$, of the distribution of $\pi\left(\boldsymbol{Z}_{n}\right) / \sqrt{n}$ conditioned on the event $\left\{\boldsymbol{Z}_{n} \neq \mathbf{0}, \boldsymbol{Z}_{0}=\boldsymbol{e}_{i}\right\}$ exists, and the Fourier transform of $\alpha$ is, for all $s \in E^{*}$ such that $\langle\boldsymbol{v}, \boldsymbol{s}\rangle=0$,

$$
\int_{E / \mathbb{R} \boldsymbol{v}} \mathrm{e}^{\mathrm{i}\langle\boldsymbol{u}, \boldsymbol{s}\rangle} \alpha(\mathrm{d} \boldsymbol{u})=\lim _{n \rightarrow \infty} \mathrm{E}\left(\mathrm{e}^{\mathrm{i}\left\langle Z_{n} / \sqrt{n}, \boldsymbol{s}\right\rangle} \mid \boldsymbol{Z}_{n} \neq \mathbf{0}, \boldsymbol{Z}_{0}=\boldsymbol{e}_{i}\right)=\left(1+\langle\boldsymbol{w}, \boldsymbol{\mu}\rangle\left\langle\boldsymbol{v}, \boldsymbol{s}^{2}\right\rangle\right)^{-1},
$$

where $\boldsymbol{s}^{2}$ is the vector in $E^{*}$ obtained by squaring the coordinates of $\boldsymbol{s}$. The limit does not depend on $i=1, \ldots, k$.

Remark 5. For a general multitype process, part 2 of Theorem 2 can be found in Athreya and Ney (1972, p. 191). Here, for a rational multitype generating function, we have an elementary proof and a rather explicit description, also given in Pollak (1974, Equation (4.4)). The Fourier transform of $\alpha$ shows that any of its projections on a real line is a bilateral exponential distribution. However, it is worthwhile describing $\alpha(\mathrm{d} \boldsymbol{u})$ in some detail. Observe that $s \mapsto Q(\boldsymbol{s})=\langle\boldsymbol{w}, \boldsymbol{\mu}\rangle\left\langle\boldsymbol{v}, \boldsymbol{s}^{2}\right\rangle$ is a positive-definite quadratic form on the subspace $(\mathbb{R} \boldsymbol{v})^{\perp}$ of $E^{*}$, which is the orthogonal complement of $\mathbb{R} \boldsymbol{v}$ as well as the dual space of $E / \mathbb{R} \boldsymbol{v}$. Introduce the unique symmetric linear map $a$ from $(\mathbb{R} \boldsymbol{v})^{\perp}$ to $E / \mathbb{R} \boldsymbol{v}$ defined by $\langle a(\boldsymbol{s}), \boldsymbol{s}\rangle=Q(\boldsymbol{s})$ (it is easy to prove that $\left\langle a(\boldsymbol{s}), \boldsymbol{s}^{\prime}\right\rangle=\langle\boldsymbol{w}, \boldsymbol{\mu}\rangle\left\langle\boldsymbol{v}, \boldsymbol{s} \boldsymbol{s}^{\prime}\right\rangle$ where $\boldsymbol{s} \boldsymbol{s}^{\prime}$ is simply the vector of $E^{*}$ obtained by coordinatewise multiplication). We now write

$$
\begin{aligned}
(1+Q(\boldsymbol{s}))^{-1} & =\int_{0}^{\infty} \mathrm{e}^{-\sigma^{2} / 2} \mathrm{e}^{-\left(\sigma^{2} / 2\right)\langle a(\boldsymbol{s}), \boldsymbol{s}\rangle} \sigma \mathrm{d} \sigma \\
& =\frac{C}{(2 \pi)^{(k-1) / 2}} \int_{E / \mathbb{R} \boldsymbol{v}} \mathrm{e}^{\mathrm{i}\langle\boldsymbol{u}, \boldsymbol{s}\rangle}\left(\int_{0}^{\infty} \mathrm{e}^{-\sigma^{2} / 2} \mathrm{e}^{-\left\langle\boldsymbol{u}, a^{-1}(\boldsymbol{u})\right\rangle / 2 \sigma^{2}} \frac{\mathrm{d} \sigma}{\sigma^{k-2}}\right) \mathrm{d} \boldsymbol{u},
\end{aligned}
$$

where $C$ is a constant depending both on the choice of Lebesgue measure $\mathrm{d} \boldsymbol{u}$ on $E / \mathbb{R} \boldsymbol{v}$ and on $a$. Actually if $E / \mathbb{R} \boldsymbol{v}$ has the Euclidean structure induced by the squared norm $\|\boldsymbol{u}\|^{2}=\left\langle\boldsymbol{u}, a^{-1}(\boldsymbol{u})\right\rangle$ 
and if $\mathrm{d} \boldsymbol{u}$ is the canonical Lebesgue measure associated with this Euclidean space, then $C=1$. Computing the last integral yields

$$
\alpha(\mathrm{d} \boldsymbol{u})=\frac{C}{2(2 \pi)^{(k-1) / 2}}\left\langle\boldsymbol{u}, a^{-1}(\boldsymbol{u})\right\rangle^{(k-2) / 4} K_{(k-2) / 2}\left(\sqrt{\left\langle\boldsymbol{u}, a^{-1}(\boldsymbol{u})\right\rangle}\right) \mathrm{d} \boldsymbol{u},
$$

where $K_{p}(\lambda)=\int_{0}^{\infty} \mathrm{e}^{-(\lambda / 2)(t+1 / t)} t^{p-1} \mathrm{~d} t$ is a modified Bessel function of the third kind.

The normalization in part 3 of Theorem 2 was introduced by Ney; see Athreya and Ney (1972, p. 192, Theorem 2).

Proof of Theorem 2. 1. The fact that $\boldsymbol{Z}_{n}$ tends to $\mathbf{0}$ is classical.

2. This is also a classical fact, but for a fractional linear birth law the proof is rather simple. In (14) we replace $s$ by

$$
\mathrm{e}^{-\boldsymbol{s} / n}=\mathbf{1}-\frac{\boldsymbol{s}}{n}+\frac{\boldsymbol{\varepsilon}(n)}{n},
$$

where $\varepsilon(n) \in E^{*}$ tends to $\mathbf{0}$. We now use (10), (11), and (4) and we apply (8) to the case $\rho=1$. Passing to the limit gives the result.

3. The proof of part 3 is similar. In order to avoid the introduction of an artificial Euclidean structure on $E$, we consider the random variable $\pi\left(\boldsymbol{Z}_{n}\right) / \sqrt{n}$ with values in the quotient space $H=E / \mathbb{R} \boldsymbol{v}$. We look for its Fourier transform, which is a function on the dual space $H^{*}=$ $(E / \mathbb{R} \boldsymbol{v})^{*}$, which in turn is the subspace of vectors $s \in E^{*}$ such that $\langle\boldsymbol{v}, \boldsymbol{s}\rangle=0$. In (14) we replace $s$ by

$$
\mathrm{e}^{\mathrm{i} s / \sqrt{n}}=\mathbf{1}+\frac{\mathrm{i} \boldsymbol{s}}{\sqrt{n}}-\frac{\boldsymbol{s}^{2}}{n}+\frac{\boldsymbol{\varepsilon}(n)}{n},
$$

where $\boldsymbol{\varepsilon}(n) \in E^{*}$ tends to $\mathbf{0}$ and $\langle\boldsymbol{v}, \boldsymbol{s}\rangle=0$. We again use (10), (11), and (4) and apply (8) to the case $\rho=1$. Passing to the limit then gives the final result.

\section{The case $\rho>1$}

Theorem 3. Let $Z$ be a branching process governed by a regular fractional linear birth law parameterized by $\boldsymbol{t} \in T$ and by the mean $\boldsymbol{M}$. Let us assume that $\rho>1$. Respectively denote by $\boldsymbol{\mu}$ and $\boldsymbol{v}$ the right and left eigenvectors of $\boldsymbol{M}$ for $\rho$ normalized such that $\langle\boldsymbol{v}, \boldsymbol{\mu}\rangle=\langle\boldsymbol{v}, \mathbf{1}\rangle=1$.

1. Denote by $\boldsymbol{q} \in E^{*}$ the column vector defined by $q_{i}=\operatorname{Pr}\left(\lim _{n \rightarrow \infty} \boldsymbol{Z}_{n}=\mathbf{0} \mid \boldsymbol{Z}_{0}=\boldsymbol{e}_{i}\right)$, namely the vector of extinction probabilities. Then $\boldsymbol{q}$ is characterized by the fact that $\mathbf{1}-\boldsymbol{q}$ is the eigenvector of $\boldsymbol{M}$ for the eigenvalue $\rho$ such that $1-\langle\boldsymbol{t}, \boldsymbol{q}\rangle=t_{0} \rho$. Furthermore, $(\boldsymbol{q}, 1)^{\top}$ is an eigenvector of $\boldsymbol{G}$ for the eigenvalue $\rho t_{0}$ and, with the notation $\boldsymbol{w}=\boldsymbol{t} / t_{0}$, we have

$$
\boldsymbol{q}=\mathbf{1}-\frac{(\rho-1) \boldsymbol{\mu}}{\langle\boldsymbol{w}, \boldsymbol{\mu}\rangle}
$$

2. The limit $\boldsymbol{W}=\lim _{n \rightarrow \infty} \boldsymbol{Z}_{n} / \rho^{n}$ exists almost surely, and its distribution for $\boldsymbol{Z}_{0}=\boldsymbol{e}_{i}$ is a mixture of a Dirac mass at $\mathbf{0}$ and an exponential distribution concentrated on the line $\mathbb{R} \boldsymbol{v}$. More specifically, its Laplace transform is

$$
\mathrm{E}\left(\mathrm{e}^{-\langle\boldsymbol{W}, \boldsymbol{s}\rangle} \mid \boldsymbol{Z}_{0}=\boldsymbol{e}_{i}\right)=q_{i}+\left(1-q_{i}\right) \frac{\rho-1}{\rho-1+\langle\boldsymbol{w}, \boldsymbol{\mu}\rangle\langle\boldsymbol{v}, \boldsymbol{s}\rangle} .
$$


3. Denote by $\pi$ the canonical projection of $E$ on the quotient space $E / \mathbb{R} \boldsymbol{v}$. Then the limit, $\beta_{i}$, of the distribution of $\pi\left(\boldsymbol{Z}_{n}\right) / \rho^{n / 2}$ conditioned on $\boldsymbol{Z}_{0}=\boldsymbol{e}_{i}$ is given by its Fourier transform, defined for all $\boldsymbol{s} \in E^{*}$ such that $\langle\boldsymbol{v}, \boldsymbol{s}\rangle=0$ by

$$
\lim _{n \rightarrow \infty}\left(\mathrm{E}\left(\mathrm{e}^{\mathrm{i}\left\langle\pi\left(\boldsymbol{Z}_{n}\right) / \rho^{n / 2}, \boldsymbol{s}\right\rangle} \mid \boldsymbol{Z}_{0}=\boldsymbol{e}_{i}\right)\right)_{i=1}^{k}=\mathbf{1}-\frac{(\rho-1) \boldsymbol{\mu}}{\langle\boldsymbol{w}, \boldsymbol{\mu}\rangle}+\frac{(\rho-1) \boldsymbol{\mu}}{\rho-1+\langle\boldsymbol{w}, \boldsymbol{\mu}\rangle\left\langle\boldsymbol{v}, \boldsymbol{s}^{2}\right\rangle} .
$$

Remark 6. The remarks, following the statement of Theorem 2, about a distribution concentrated on $E / \mathbb{R} \boldsymbol{v}$ are still applicable. The probability $\beta_{i}$ is a mixture of the image of the dilation $x \mapsto(\rho-1)^{1 / 2} x$ of the probability $\alpha$ occurring in Theorem 2 with an atom at $\mathbf{0}$ of mass $q_{i}$. Also, note that parts 1 and 2 of Theorem 3 were given in different forms in Pollak (1974, Equations 3.1 and 4.3).

Example 2. $(k=2$.) The probability of extinction can be explicitly computed. For $k=2$ we are given three positive numbers, $t_{0}, t_{1}$, and $t_{2}$, such that $t_{0}+t_{1}+t_{2}=1$, and the mean matrix

$$
\boldsymbol{M}=\left(\begin{array}{cc}
m_{1} & m_{12} \\
m_{21} & m_{2}
\end{array}\right)
$$

We compute $\boldsymbol{q}=\left(q_{1}, q_{2}\right)^{\top}$. For simplicity, we write $\Delta=\left(m_{1}-m_{2}\right)^{2}+4 m_{12} m_{21}$. Thus, the largest eigenvalue of $\boldsymbol{M}$ is $\rho=\frac{1}{2}\left(m_{1}+m_{2}+\sqrt{\Delta}\right)$. We have $\rho>1$ if and only if $1+\operatorname{det} \boldsymbol{M}<\operatorname{trace} \boldsymbol{M}$. The corresponding eigenvector, $\left(v_{1}, v_{2}\right)=\left(1-q_{1}, 1-q_{2}\right)$, therefore satisfies the linear equation

$$
\left(\rho-m_{1}\right) v_{1}=m_{12} v_{2} .
$$

Furthermore, the condition $1-\langle\boldsymbol{t}, \boldsymbol{q}\rangle=t_{0} \rho$ becomes

$$
t_{1} v_{1}+t_{2} v_{2}=t_{0}(\rho-1)
$$

and $v_{1}$ and $v_{2}$ are obtained by solving a linear system.

Proof of Theorem 3. The vector $\boldsymbol{q}=\left(q_{1}, \ldots, q_{k}\right)^{\top}$ of extinction probabilities (see Harris (1963, p. 41)) belonging to $E^{*}$ satisfies

$$
G\left(\begin{array}{l}
\boldsymbol{q} \\
1
\end{array}\right)=\lambda\left(\begin{array}{l}
\boldsymbol{q} \\
1
\end{array}\right)
$$

for some real number $\lambda \neq 0$; that is, $(\boldsymbol{q}, 1)^{\top}$ is an eigenvector of $\boldsymbol{G}$. To see this, we observe that $h_{\boldsymbol{G}}(\boldsymbol{q})=\boldsymbol{q}$ implies (16) with $\lambda=\langle\boldsymbol{c}, \boldsymbol{q}\rangle+d$. Furthermore, since this birth law is regular, it is positively regular in the sense of Harris (1963, p. 38). The largest positive eigenvalue, $\rho$, of $M$ exists and is simple and all complex eigenvalues of $M$ have modulus less than $\rho$. This implies that

$$
t_{0} \boldsymbol{M} \boldsymbol{q}-\mathbf{1} \boldsymbol{t}^{\top} \boldsymbol{q}+\left(\boldsymbol{I}_{k}-t_{0} \boldsymbol{M}\right) \mathbf{1}=\lambda \boldsymbol{q},
$$

which, with the above value of $\lambda$, is equivalent to

$$
t_{0} \boldsymbol{M}(\mathbf{1}-\boldsymbol{q})=\lambda(\mathbf{1}-\boldsymbol{q})
$$

Now, from a classical result (Harris (1963, Chapter 2, Theorem 8.1), $\mathbf{1}-\boldsymbol{q}$ has strictly positive components. The Perron-Frobenius theorem (Harris (1963, p. 37)) implies that $\lambda=t_{0} \rho$ and that the dimension of the eigenspace of $t_{0} \boldsymbol{M}$ associated with it is one. 
For the second part of the theorem, the existence of the almost-sure limit $\boldsymbol{W}$ of $\boldsymbol{Z}_{n} / \rho^{n}$ is classical. To find the distribution of $\boldsymbol{W}$, we look for the limit of the sequence

$$
\left(\mathrm{E}\left(\mathrm{e}^{-\left\langle\boldsymbol{s}, \boldsymbol{Z}_{n} / \rho^{n}\right\rangle} \mid \boldsymbol{Z}_{0}=\boldsymbol{e}_{i}\right)\right)_{i=1}^{k}=h_{\boldsymbol{G}^{n}}\left(\mathrm{e}^{-\boldsymbol{s} / \rho^{n}}\right) \quad \text { as } n \rightarrow \infty .
$$

Now we replace $\boldsymbol{s}$ in (9) by $\mathrm{e}^{-\boldsymbol{s} / \rho^{n}}=\mathbf{1}-\rho^{-n} \boldsymbol{s}+\rho^{-n} \boldsymbol{\varepsilon}(n)$, where the vector $\boldsymbol{\varepsilon}(n)$ tends to $\mathbf{0}$ as $n$ tends to $\infty$. We then use (5) and (8) to obtain (15) by an easy calculation. from

The proof of the third part is quite similar to that of the third part of Theorem 2, starting

$$
\mathrm{e}^{\mathrm{i} s / \rho^{n / 2}}=\mathbf{1}+\frac{\mathrm{i} \boldsymbol{s}}{\rho^{n / 2}}-\frac{\boldsymbol{s}^{2}}{\rho^{n}}+\frac{\boldsymbol{\varepsilon}(n)}{\rho^{n}} .
$$

The calculations are standard and use the fact that $\boldsymbol{L}_{n}=\boldsymbol{M}^{n}-\rho^{n} \boldsymbol{\mu} \otimes \boldsymbol{v}$ has a spectral norm, $\rho_{0}^{n}$, which is less than or equal to 1 , from Proposition 5. Thus, $\rho^{-n / 2} \boldsymbol{L}_{n}$ tends to $\mathbf{0}$, which leads to the result.

\section{Total progeny}

For $\rho \leq 1$, the multitype branching process $Z=\left(\boldsymbol{Z}_{n}\right)_{n \geq 0}$ governed by a regular fractional linear birth law satisfies $\lim _{n \rightarrow \infty} \boldsymbol{Z}_{n}=\mathbf{0}$, and the total progeny,

$$
S=\sum_{n=0}^{\infty} \boldsymbol{Z}_{n}
$$

is a finite vector. When $\boldsymbol{Z}_{0}=\boldsymbol{z}_{0} \in \mathbb{N}^{k}$ is not random, we write $\boldsymbol{S}=\boldsymbol{S}_{z_{0}}$. We also write $g_{j}(\boldsymbol{s})=\mathrm{E}\left(\boldsymbol{s}^{\boldsymbol{S}_{\boldsymbol{e}_{j}}}\right)$ and $\boldsymbol{g}(\boldsymbol{s})=\left(g_{1}(\boldsymbol{s}), \ldots, g_{k}(\boldsymbol{s})\right)^{\top}$. It is then easy to see that, as in the onedimensional case,

$$
\boldsymbol{g}(\boldsymbol{s})=\operatorname{diag}(\boldsymbol{s}) h_{\boldsymbol{G}}(\boldsymbol{g}(\boldsymbol{s})) .
$$

Here again this is equivalent to saying that there exists a $\lambda \neq 0$ such that

$$
\left(\begin{array}{cc}
\operatorname{diag}(s) A & \operatorname{diag}(s) b \\
c & d
\end{array}\right)\left(\begin{array}{c}
g(s) \\
1
\end{array}\right)=\lambda\left(\begin{array}{c}
g(s) \\
1
\end{array}\right) .
$$

Here the eigenvalue $\lambda$ must equal $\langle\boldsymbol{c}, \boldsymbol{g}(\boldsymbol{s})\rangle+d$. However, while for $k=1$ an explicit solution for $g$ is easily obtained from

$$
g(s)=\frac{1-s(r-t)-\left[(1-s(r-t))^{2}-4 s(1-r) t\right]^{1 / 2}}{2 t},
$$

for $k=2$ the explicit solution is too complex to be displayed here, since the eigenvalue $\lambda$ has the form

$$
p_{1}+\left(p_{3}+p_{6}^{1 / 2}\right)^{1 / 3}+\left(p_{3}-p_{6}^{1 / 2}\right)^{1 / 3}
$$

where $p_{j}$ is an inhomogeneous polynomial in $s_{1}$ and $s_{2}$ of degree $j$.

\section{Imbedding in a continuous semigroup for $k \geq 2$}

For $k=1$, consider the fractional birth law governed by the generating function

$$
f(s)=h_{G}(s)=1-r+(1-t) \frac{r s}{1-r s}=\frac{(r-t) s+1-r}{1-t s},
$$


where, in the notation of Proposition 2,

$$
\boldsymbol{G} \equiv \boldsymbol{G}(t, r)=\left(\begin{array}{cc}
r-t & 1-r \\
-t & 1
\end{array}\right)=\left(\begin{array}{cc}
(1-t) m-t & 1-(1-t) m \\
-t & 1
\end{array}\right)
$$

with $m=r /(1-t)$ as usual. If $\alpha>0$ then the fractional power of the matrix $\boldsymbol{G}$ is

$$
\boldsymbol{G}^{\alpha}=\left\{\begin{array}{cc}
\frac{1}{t_{0}-r}\left(\begin{array}{cc}
r_{0} r^{\alpha}-t t_{0}^{\alpha} & r_{0}\left(t_{0}^{\alpha}-r^{\alpha}\right) \\
-t\left(t_{0}^{\alpha}-r^{\alpha}\right) & -t r^{\alpha}+r_{0} t_{0}^{\alpha}
\end{array}\right) & \text { for } m \neq 1 \\
r^{\alpha-1}\left(\begin{array}{cc}
1-(\alpha+1) t & \alpha t \\
-\alpha t & 1+(\alpha-1) t
\end{array}\right) & \text { for } m=1
\end{array}\right.
$$

It can indeed be checked both that $\alpha \mapsto G^{\alpha}$ is continuous on $[0, \infty)$ with the convention $\boldsymbol{G}^{0}=\boldsymbol{I}_{2}$ and, by inspection, that $\boldsymbol{G}^{\alpha} \boldsymbol{G}^{\beta}=\boldsymbol{G}^{\alpha+\beta}$.

This formula can be guessed, for positive integers $\alpha=n$, from the results of Proposition 3 . The fractional linear function $h_{\boldsymbol{G}^{n}}=h_{\boldsymbol{G}} \circ \cdots \circ h_{\boldsymbol{G}}$ is a generating function. Since $h_{\boldsymbol{M}}=h_{\boldsymbol{M}^{\prime}}$ implies the existence of a $\lambda$ such that $\boldsymbol{M}^{\prime}=\lambda \boldsymbol{M}$, there must exist $t^{(n)} \in(0,1), r^{(n)} \in(0,1]$, and $\lambda^{(n)}>0$ such that $\boldsymbol{G}^{n}=\lambda_{n} \boldsymbol{G}\left(t^{(n)}, r^{(n)}\right)$. By applying $w^{(n)}=w\left(1+\cdots+m^{n-1}\right)$ and the identities (7), for $m \neq 1$ we find that

$$
\begin{aligned}
w^{(n)}=\frac{t^{(n)}}{t_{0}^{(n)}} & =\frac{t t^{n}-t r^{n}}{t_{0}^{n+1}-r t_{0}^{n}}, & \lambda^{(n)} & =\frac{-t r^{n}+r_{0} t_{0}^{n}}{t_{0}-r}, \\
t^{(n)} & =\frac{\left(t_{0}^{n}-r^{n}\right) t}{-t r^{n}+r_{0} t_{0}^{n}}, & r^{(n)} & =\left(1-t^{(n)}\right) m^{n} .
\end{aligned}
$$

If $m=1$, that is, if $r=1-t=t_{0}$, similar calculations hold with

$$
\begin{aligned}
w^{(n)} & =n \frac{t}{t_{0}}, & \lambda^{(n)} & =t_{0}^{n-1}(1+(n-1) t), \\
t^{(n)} & =\frac{n t}{1+(n-1) t}, & r^{(n)} & =\frac{t_{0}}{1+(n-1) t} .
\end{aligned}
$$

Thus, (17) and (18) hold for any positive integer $\alpha=n$.

Now we define $\lambda^{(\alpha)}, t^{(\alpha)}$, and $r^{(\alpha)}$ for the continuous parameter $\alpha \geq 0$ by simply replacing $n$ by $\alpha$ in (19) (for $m \neq 1$ ) and in (20) (for $m=1$ ). We observe that $\lambda^{(\alpha)}>0, t^{(\alpha)} \in(0,1)$, and $r^{(\alpha)} \in(0,1]$ still hold; therefore, $\boldsymbol{G}_{\alpha}=\boldsymbol{G}\left(t^{(\alpha)}, r^{(\alpha)}\right)$ makes sense. Note that $\boldsymbol{G}^{\alpha}$ defined by (17) and (18) satisfies $\boldsymbol{G}^{\alpha}=\lambda^{(\alpha)} \boldsymbol{G}_{\alpha}$. Checking that $\boldsymbol{G}^{\alpha}$ satisfies $\boldsymbol{G}^{\alpha} \boldsymbol{G}^{\beta}=\boldsymbol{G}^{\alpha+\beta}$ for all $\alpha, \beta \geq 0$ is a tedious task. The reward is that, for

$$
h_{\boldsymbol{G}_{\alpha}}(s)=\frac{\left(r^{(\alpha)}-t^{(\alpha)}\right) s+1-r^{(\alpha)}}{1-t^{(\alpha)} s},
$$

we have $h_{\boldsymbol{G}_{\alpha}} \circ h_{\boldsymbol{G}_{\beta}}(s)=h_{\boldsymbol{G}_{\alpha+\beta}}(s)$. Furthermore, $\lim _{\alpha \rightarrow 0} h_{\boldsymbol{G}_{\alpha}}(s)=s$. Thus, $\alpha \mapsto h_{\boldsymbol{G}_{\alpha}}$ provides a continuous imbedding of the transition matrix of the Markov chain $\left(Z_{n}\right)_{n \geq 0}$ into a continuous semigroup. The imbedding of a discrete branching process into a continuous one has a venerable history. Other than Harris (1963, Chapter V, Section 5) and Athreya and Ney (1972, Chapter III, Section 12), we cite Karlin and McGregor (1968a), (1968b), Goryainov (1993), (1995), (2002), and Grey (1975). We thank the referee for these references. Most of these authors mention the fractional linear case and give its characteristic properties for $k=1$. 
We might wonder whether a similar situation holds for $k>1$. As we are going to see for $k=2$, the answer is 'not in general'. There are multitype branching processes with a fractional birth law which are not imbeddable. In (7) we in general cannot simply replace the positive integer $n$ by the positive real number $\alpha$ as we can for $k=1$. To see this, we first state without proof a simple proposition giving a necessary condition for imbeddability, and make an elementary remark about $2 \times 2$ matrices.

Proposition 7. Let $\boldsymbol{G}$ be the $(k+1) \times(k+1)$ matrix associated with a regular birth law with mean matrix $\boldsymbol{M}$, such that the process is imbeddable. Then the following statements hold.

1. There exists a continuous mapping $\alpha \mapsto \boldsymbol{M}_{\alpha}$, from $[0, \infty)$ to the matrices with positive entries, such that $\boldsymbol{M}_{\alpha+\beta}=\boldsymbol{M}_{\alpha} \boldsymbol{M}_{\beta}, \boldsymbol{M}_{1}=\boldsymbol{M}$, and $\lim _{\alpha \rightarrow 0} \boldsymbol{M}_{\alpha}=\boldsymbol{I}_{k}$.

2. There exists a $k \times k$ matrix $\boldsymbol{Q}$ such that $\boldsymbol{M}=\mathrm{e}^{\boldsymbol{Q}}$, where $\boldsymbol{Q}$ has only nonpositive diagonal elements and the nondiagonal elements of $\boldsymbol{Q}$ are positive.

Remark 7. If $\boldsymbol{M}$ has rank 1, say $\boldsymbol{M}=\boldsymbol{a} \otimes \boldsymbol{b}$ where $\boldsymbol{a}$ and $\boldsymbol{b}$ are vectors with positive components, then $\boldsymbol{M}_{\alpha}=\langle\boldsymbol{a}, \boldsymbol{b}\rangle^{\alpha-1} \boldsymbol{M}$ satisfies $\boldsymbol{M}_{\alpha+\beta}=\boldsymbol{M}_{\alpha} \boldsymbol{M}_{\beta}$ and $\boldsymbol{M}_{1}=\boldsymbol{M}$, but $\lim _{\alpha \rightarrow 0} \boldsymbol{M}_{\alpha} \neq \boldsymbol{I}_{k}$. In general, note that statement 2 of the previous proposition implies that $\boldsymbol{M}=\mathrm{e}^{\boldsymbol{Q}}$ is invertible.

Proposition 8. Let $a, b \in(0,1)$. There then exist $\alpha, \beta \geq 0$ such that

$$
\left(\begin{array}{cc}
a & 1-a \\
1-b & b
\end{array}\right)=\exp \left(\begin{array}{cc}
-\alpha & \alpha \\
\beta & -\beta
\end{array}\right)
$$

if and only if $a+b>1$.

We now show from these two propositions that there exists a fractional birth law for $k=2$ which is not imbeddable, while $\boldsymbol{M}$ is invertible. For this, we take $\left(w_{1}, w_{2}\right)=\boldsymbol{t} / t_{0}$ with $w_{1}+w_{2}<1$. The mean vectors $\boldsymbol{m}$ which are compatible with $\boldsymbol{w}$ are in the triangle with vertices $(0,0),\left(w_{1}+1, w_{2}\right)$, and $\left(w_{1}, w_{2}+1\right)$ (see Remark 1$)$. Thus, we can find two such vectors, of respective forms $(a, 1-a)$ and $(1-b, b)$. Since they lie in the prescribed triangle, they satisfy

$$
w_{1} \leq a \leq 1-w_{2}, \quad w_{2} \leq b \leq 1-w_{1} .
$$

Taking, for instance, $\varepsilon<\frac{1}{2}\left(1-w_{1}-w_{2}\right)$ and $a=w_{1}+\varepsilon, b=w_{2}+\varepsilon$ will give $a+b<1$. Thus, the fractional linear birth law associated with $\boldsymbol{M}$ and $\boldsymbol{w}$ does exist, while from Propositions 7 and 8 it is not imbeddable. Choosing $\varepsilon$ properly gives det $\boldsymbol{M} \neq 0$.

\section{Acknowledgement}

The first author thanks NSERC, whose generous support made this collaboration possible.

\section{References}

Athreya, K. B. And Ney, P. E. (1972). Branching Processes. Springer, Berlin.

Goryainov, V. V. (1993). Fractional iteration of probability-generating functions and imbedding discrete branching processes into continuous processes. Mat. Sb. 184, 55-74 (in Russian). English translation: Russian Acad. Sci. Sb. Math. 79, 47-61, 1994.

Goryainov, V. V. (1995). Semigroups of probability generating function, and infinitely splittable functions. Theory Random Process. V.1 17, 2-9.

Goryainov, V. V. (2002). Koenigs function and fractional iterates of probability generating functions. Sb. Math. 193, 1009-1025.

Grey, D. R. (1975). Two necessary conditions for embeddability of a Galton-Watson branching process. Math. Proc. Camb. Phil. Soc. 78, 339-343. 
Harris, T. E. (1963). The Theory of Branching Processes. Springer, Berlin.

Karlin, S. AND McGregor, J. (1968a). Embeddability of discrete time simple branching processes into continuous time branching processes. Trans. Amer. Math. Soc. 132, 115-136.

KARLIN, S. AND MCGREGOR, J. (1968b). Embedding iterates of analytic functions with two fixed points into continuous groups. Trans. Amer. Math. Soc. 132, 137-145.

PollaK, E. (1974). Survival probabilities and extinction times for some multitype branching processes. Adv. Appl. Prob. 6, 446-462.

Toulouse, P. S. (1999). Thèmes de Probabilités et Statistique. Dunod, Paris. 